\title{
Comparison of Dual-Channel Supply Chain Structures: E-Commerce Platform as Different Roles
}

\author{
Cong Wang, ${ }^{1}$ De-li Yang, ${ }^{1}$ and Zhao Wang ${ }^{2}$ \\ ${ }^{1}$ Faculty of Management and Economics, Dalian University of Technology, Dalian 116024, China \\ ${ }^{2}$ Bank of Dalian Co. Ltd., Dalian 116001, China \\ Correspondence should be addressed to Cong Wang; congcong830702@163.com
}

Received 22 February 2016; Revised 26 July 2016; Accepted 7 August 2016

Academic Editor: Laura Gardini

Copyright (c) 2016 Cong Wang et al. This is an open access article distributed under the Creative Commons Attribution License, which permits unrestricted use, distribution, and reproduction in any medium, provided the original work is properly cited.

E-commerce platforms can act as an e-tailer or a third-party intermediary that charge a commission and allow manufacturers to sell products on their platform directly. These two forms of dual-channel supply chain structures have different impacts on decisions and profits of manufacturers and traditional retailers. This paper establishes different Stackelberg game models for both dual-channel supply chain structures and achieves the equilibrium results. The results state that both structures can increase the consumer surplus and social welfare. More importantly, when the platform acts as an e-tailer, the manufacturer and the traditional retailer can form a "win-win" situation. Nevertheless, when the platform acts as a third-party intermediary allowing manufacturers to sell products on the platform directly, it is bound to harm the interests of the traditional retailer.

\section{Introduction}

With the development of e-commerce, many manufacturers, such as IBM and Nike, have added a direct e-channel to adopt dual-channel strategy. In addition, many researchers have verified that the dual-channel strategy can make more profit for manufacturers in some conditions (e.g., $[1,2]$ ). However, most of the studies are based on the platforms that are owned by manufacturers. In practice, the majority of manufacturers do not have the ability to build e-commerce platforms or their platforms cannot compete with prominent e-commerce platforms. Hence, these manufacturers have to use a third-party e-commerce platform when they adopt the dual-channel strategy. For example, IMB also sells on Amazon even though it has its own sales website. Therefore, there is a theoretical gap in the dual-channel strategy whose electronic channel is based on a third-party platform.

Recently, the large e-commerce platforms such as Amazon and Sears have broken through the traditional reselling format and embraced the agency model [3]. It means that a platform owner can either act as an e-tailer or an agent who authorizes manufacturers to sell products on the platform directly. Due to funding constraints and other reasons, the platform enterprise only chooses some kinds of products to sell by itself but earns the commission as an agent with vast majority of products. This study is motivated by the following two questions:

(1) If the platform enterprise does not choose the product to sell by itself, will the manufacturer accept the commission policy to join in the platform and sell products directly or only reserve the traditional channel?

(2) How do the different roles of the platform enterprise influence the operating decisions and business performances of the manufacturers and traditional retailers? And what about the consumer surplus and social welfare?

This study provides the performance comparisons of supply chain members between the two different dual-channel supply chain structures and the single channel structure. We use the game theory approach to develop two dual-channel supply chain structures where the e-commerce owner can act as an e-tailer ( $E$ Structure) or an agent authorizing the manufacturer to sell products on the platform directly ( $P$ Structure). In order to get some implications to promote 
the development of e-commerce, we also compare the consumer surplus and social welfare in different supply chain structures through numerical experiments.

The rest of this paper is organized as follows. After summarizing the related literature in Section 2, two forms of dual-channel supply chain structurers based on thirdparty e-commerce platforms are introduced in Section 3. The equilibrium results in different supply chain structures are provided in Section 4. After the comparison analysis of the equilibrium results, the consumer surplus and the social welfare are calculated in Section 5. Section 6 describes the additional numerical experiments. The conclusion and some management implications are presented at the end of this paper.

\section{Literature Review}

This paper focuses on dual-channel supply chain structures based on third-party e-commerce platform. Thus, the related literature includes the areas of dual-channel management and e-commerce platform.

The literature about dual-channel management mainly involves channel competition, pricing strategy, and dualchannel supply chain coordination. A large amount of literature focuses on channel competition and pricing strategy of the dual-channel supply chain. Chiang et al. [4] demonstrated that the manufacturer would like to add a direct channel to the existing traditional retail channel and reported a zone where the manufacturer and the traditional retailer can be both better off than in the single channel structure. Croom [5] reported that operating in dual-channel supply chain has become an effective means of competition between supply chains members and different supply chains with the development of e-commerce. Baye [6] provided insights into the competition between a pure play e-tailer and a bricksand-clicks e-tailer and found out that the pure play e-tailer always had a lower equilibrium price. Park and Keh [7] pointed out that the profits of the manufacturer and the whole supply chain will be better but the traditional retailer's profit will be worse when the manufacturer adds a direct channel. $\mathrm{Lu}$ and Liu [8] discussed the influence on the supply chain for the manufacturer opening the electronic channel and put forward the necessary conditions for the manufacturer. Li et al. $[9,10]$ discussed the pricing strategy of the dualchannel supply chain for small and medium sized enterprises and supply chain with a risk-averse retailer, respectively. The literature about dual-channel supply chain coordination is also quite comprehensive. A lot of the observed papers focus on the coordination of dual-channel supply chain by different contracts [11-14]. And there are some other cooperation strategies to get a win-win state for the manufacturer and the retailer, such as incentive schemes [15], service cooperation [16], and advertising cooperation [17].

The literature about e-commerce platform is basically focused on the platform pricing, mainly paying attention to fixed fees, proportional fees, or two-part tariffs [18-20]. Muthers and Wismer [21] demonstrated that proportional fees offer more advantages to avoid the risk of moral hazard and attract more participants in comparison with two-part tariffs. Wang and Wright [22] extended the area of the proportional fees with price discrimination. The literature about the influence of e-commerce platforms on supply chain has also been concerned by many scholars in recent years. The most relevant literature to this paper is whether the role mode of the platform in supply chain is a "merchant" mode or a "two-sided platform." Hagiu [23] demonstrated the choice of the market intermediation between a "merchant" mode and a "two-sided platform" mode with different influence factors. Abhishek et al. [3] provided that the mode choice of the platform enterprise between agency selling and reselling was relevant to the effect of sales in the electronic channel on the demand in the traditional channel. Mantin et al. [24] found out that e-tailer's power of bargaining with the manufacturer could be increased after he built the platform and it benefited the consumers, but it would decrease the consumers' benefits if the power is too big. Jiang et al. [25] used "Mid Tail" theory to analyze the mode selection problem of the platform e-tailer and suggested that the e-tailer might select different modes for different kinds of products.

Based on the literature above, this paper assumes that the manufacturer does not have the ability to build an ecommerce platform or his platform cannot compete with prominent e-commerce platforms. Thus, the manufacturer must add the electronic channel through a third-party ecommerce platform if he adopts a dual-channel strategy. Then, we analyze the supply chain members' operation decisions and their performance in different supply chain structures caused by different roles of the platform.

\section{Model Assumptions and Notations}

A system analyzed in this paper consists of a manufacturer, a traditional retailer, and an e-commerce platform owner. They can be composed of two kinds of supply chain structures according to the different roles of the platform owner in the supply chain. For simplicity, we describe two structures, " $E$ Structure" and " $P$ Structure," in Figure 1.

Without loss of generality, we normalize the potential market size to 1 and the marginal cost of a unit product to 0 . We let $v$ denote the perceived value of products in the traditional channel. It is uniformly distributed in the interval $[0,1]$. At the same time, we denote the perceived value of products in the electronic channel as $\delta v(0<\delta<1)$, where $\delta$ denotes the perceptual coefficient of the electronic channel coming from the consumers and it reflects the substitutability of the electronic channel to the traditional channel.

Then, we consider the demand of two channels, where the prices of the traditional channel and the electronic channel are separately $p_{R}$ and $p_{D}$. Consumers whose perceived value meets the condition $v \in\left\{v \mid v-p_{R} \geq \delta v-p_{D}, v-p_{R} \geq\right.$ $0\}$ will purchase the products from the traditional channel, and consumers whose perceived value meets the condition $v \in\left\{v \mid \delta v-p_{D}>v-p_{R}, \delta v-p_{R} \geq 0\right\}$ will purchase the product from the electronic channel. Other consumers will not purchase anything. Therefore, through simple calculations, demand functions for the traditional channel and 


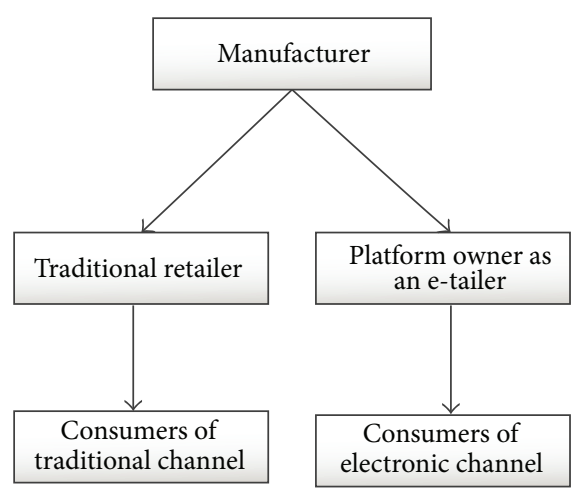

(a) E Structure

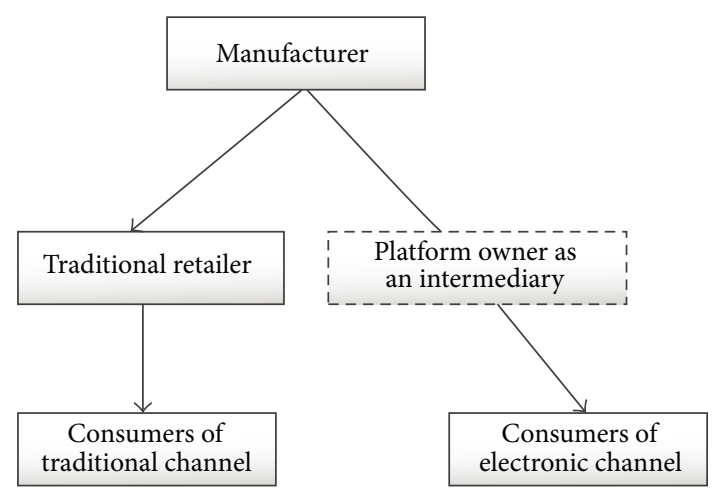

(b) P Structure

FIGURE 1: Two dual-channel supply chain structures.

the electronic channel are, respectively, as follows: $Q_{R}=$ $\left(1-\delta-p_{R}+p_{D}\right) /(1-\delta)$ and $Q_{D}=\left(\delta p_{R}-p_{D}\right) /(\delta(1-$ $\delta))$. Furthermore, based on the above demand functions, the inverse demand functions of two channels are, respectively, $p_{R}=1-Q_{R}-\delta Q_{D}$ and $p_{D}=\delta\left(1-Q_{R}-Q_{D}\right)$.

In order to express the content easily and conveniently, we use superscripts " $E$ ", " $P$," and " $N$ " to represent " $E$ Structure," " $P$ Structure," and single channel structure, and we use subscript " $M$," "TR," and " $P$ " to represent the manufacturer, the traditional retailer, and the e-commerce platform.

\section{Model Analysis}

4.1. E Structure-Platform Owner as an e-Tailer. In the E Structure, the e-commerce platform owner acts as an e-tailer. $\mathrm{He}$ and the traditional retailer sell products through their respective channels. It is assumed that the manufacturer and two channel retailers obey a two-stage Stackelberg game and the manufacturer is the leader. The sequence of events is as follows: firstly, the manufacturer determines the wholesale price of the products $w$; secondly, according to the wholesale price $w$, the traditional retailer and the e-tailer determine the supply quantity of the traditional channel $Q_{R}$ and the electronic channel $Q_{D}$, respectively.

The decision problems of the manufacturer, the traditional retailer, and the e-tailer are as follows, respectively:

$$
\begin{aligned}
& \max _{w} \Pi_{M}^{E}=w\left(Q_{R}+Q_{D}\right), \\
& \max _{Q_{R}} \Pi_{\mathrm{TR}}^{E}=\left(p_{R}-w\right) Q_{R}, \\
& \max _{Q_{D}} \Pi_{P}^{E}=\left(p_{D}-w\right) Q_{D} .
\end{aligned}
$$

According to the relation between $Q_{R}$ and $Q_{D}$ and $p_{R}$ and $p_{D}$, the objective functions of the two channel retailers can be simplified only about $w, Q_{R}$, and $Q_{D}$ as follows:

$$
\begin{aligned}
& \max _{Q_{R}} \Pi_{\mathrm{TR}}^{E}=\left(1-Q_{R}-\delta Q_{D}-w\right) Q_{R}, \\
& \max _{Q_{D}} \Pi_{P}^{E}=\left(\delta\left(1-Q_{R}-Q_{D}\right)-w\right) Q_{D} .
\end{aligned}
$$

When the wholesale price is determined, two channel retailers decide sales volume simultaneously. The second derivatives of the two channel retailers' profits about their sales volume are, respectively, $\partial^{2} \pi_{\mathrm{TR}}^{E} / \partial Q_{R}^{2}=-2<0$ and $\partial^{2} \pi_{P}^{E} / \partial Q_{D}^{2}=-2 \delta<0$, so $\pi_{\mathrm{TR}}^{E}$ and $\pi_{P}^{E}$ are both concave functions about each sales volume. Sequentially, making the first derivative of the profits of the two channel retailers about each sales volume be zero, we can get the reaction function of the sales volume about the wholesale price $w$, as follows:

$$
\begin{aligned}
Q_{R} & =\frac{2-\delta-w}{4-\delta}, \\
Q_{D} & =\frac{\delta w+\delta-2 w}{\delta(4-\delta)} .
\end{aligned}
$$

Taking the above results into the decision problem of the manufacturer, the decision function can be rewritten as follows:

$$
\max _{w} \pi_{M}^{E}=\frac{w(2-\delta-w)}{4-\delta}+\frac{w(\delta+\delta w-2 w)}{\left(4 \delta-\delta^{2}\right)} .
$$

The second derivative of $\pi_{M}^{E}$ about $w$ is $\partial^{2} \pi_{M}^{E} / \partial w^{2}=$ $-4 /(\delta(4-\delta))<0$, so $\pi_{M}^{E}$ is concave functions about the wholesale price $w$. Sequentially, making the first derivative of the profit of the manufacturer be zero, we can get the optimal wholesale price. All equilibrium results are summarized in Table 1.

4.2. P Structure-Platform Owner as an Intermediary Agency. In the $P$ Structure, the e-commerce platform owner acts as an intermediary agent. The platform enterprise charges the manufacturer a commission of $\alpha$ according to the turnover on the platform and allows the manufacturer to sell on his platform directly. The manufacturer and the traditional retailer obey a two-stage Stackelberg game and the manufacturer is the leader. The sequence of events is as follows: first, the manufacturer determines the wholesale price of the products $w$; second, according to the wholesale price $w$, the traditional retailer and the manufacturer determine the supply quantity of the traditional channel $Q_{R}$ and the electronic channel $Q_{D}$, respectively. 
TABLE 1: Equilibrium results in different structures.

\begin{tabular}{lccc}
\hline Variable & E Structure & $P$ Structure & Single channel \\
\hline$w$ & $\frac{\delta(3-\delta)}{4}$ & $\frac{8-4 \delta+\delta^{2}-2 \delta \alpha}{2(8-3 \delta+\delta \alpha)}$ & $\frac{1}{2}$ \\
$p_{R}$ & $\frac{\left(8+5 \delta-6 \delta^{2}+\delta^{3}\right)}{4(4-\delta)}$ & $\frac{(2-\delta)(6-\delta)}{2(8-3 \delta+\delta \alpha)}$ & $\frac{3}{4}$ \\
$p_{D}$ & $\frac{\delta(5-\delta)}{2(4-\delta)}$ & $\frac{\delta(6-\delta)}{2(8-3 \delta+\delta \alpha)}$ & $/$ \\
$Q_{R}$ & $\frac{\left(8-7 \delta+\delta^{2}\right)}{4(4-\delta)}$ & $\frac{2-2 \delta+\delta \alpha}{8-3 \delta+\delta \alpha}$ & $\frac{1}{4}$ \\
$Q_{D}$ & $\frac{\left(5 \delta-\delta^{2}-2\right)}{4(4-\delta)}$ & $\frac{6-\delta}{2(8-3 \delta+\delta \alpha)}$ & $/$ \\
$\pi_{M}$ & $\frac{\delta(3-\delta)^{2}}{8(4-\delta)}$ & $\frac{4+\delta^{2}-4 \delta \alpha}{4(8-3 \delta+\delta \alpha)}$ & $\frac{1}{8}$ \\
$\pi_{\mathrm{TR}}$ & $\frac{\left(\delta^{2}-7 \delta+8\right)^{2}}{16(4-\delta)^{2}}$ & $\frac{(2-2 \delta+\delta \alpha)^{2}}{(8-3 \delta+\delta \alpha)^{2}}$ & $\frac{1}{16}$ \\
$\pi_{P}$ & $\frac{\delta\left(\delta^{2}-5 \delta+2\right)^{2}}{16(4-\delta)^{2}}$ & $\frac{\delta a(\delta-6)^{2}}{4(8-3 \delta+\delta \alpha)^{2}}$ & $/$ \\
\hline
\end{tabular}

The decision problems of the manufacturer and the traditional retailer are as follows, respectively:

$$
\begin{aligned}
& \max _{w, Q_{D}} \pi_{M}^{P}=w Q_{R}+(1-\alpha) p_{D} Q_{D}, \\
& \max _{Q_{R}} \pi_{\mathrm{TR}}^{P}=\left(p_{R}-w\right) Q_{R} .
\end{aligned}
$$

According to the relation of $Q_{R}$ and $Q_{D}$ and $p_{R}$ and $p_{D}$, we include the inverse demand functions $p_{R}=1-Q_{R}-\delta Q_{D}$ and $p_{D}=\delta\left(1-Q_{R}-Q_{D}\right)$ into the objective function of the manufacturer and the traditional retailer. Then, the objective functions can be simplified only about $w, Q_{R}$, and $Q_{D}$ as follows:

$$
\begin{aligned}
& \max _{w, Q_{D}} \pi_{M}^{P}=w Q_{R}+\delta(1-\alpha)\left(1-Q_{R}-Q_{D}\right) Q_{D}, \\
& \max _{Q_{R}} \pi_{\mathrm{TR}}^{P}=\left(1-Q_{R}-\delta Q_{D}-w\right) Q_{R} .
\end{aligned}
$$

The second derivatives of the profits of the traditional retailer and the manufacturer about their sales volume are $\partial^{2} \pi_{\mathrm{TR}}^{P} / \partial Q_{R}^{P}=-2<0$ and $\partial^{2} \pi_{M}^{P} / \partial Q_{D}^{2}=-2 \delta(1-\alpha)<0$, respectively, so $\pi_{\mathrm{TR}}^{P}$ and $\pi_{M}^{P}$ are both concave functions about each sales volume. Sequentially, making the first derivative of both the profits about each sales volume be zero, we can get the reaction function of the sales volume about the wholesale price $w$ as follows:

$$
\begin{aligned}
Q_{R} & =\frac{2-\delta-2 w}{4-\delta}, \\
Q_{D} & =\frac{w+1}{4-\delta} .
\end{aligned}
$$

After taking the above results into the decision function of the manufacturer, then the decision function can be rewritten as follows:

$$
\max _{w} \pi_{M}^{P}=\frac{w(2-\delta-2 w)}{4-\delta}+\frac{\delta(1-\alpha)(1+w)^{2}}{(4-\delta)^{2}}
$$

The second derivative of $\pi_{M}^{P}$ about $w$ is $\partial^{2} \pi_{M}^{P} / \partial w^{2}=$ $-2(8-3 \delta+\delta \alpha) /(4-\delta)^{2}<0$, so $\pi_{M}^{P}$ is concave functions about wholesale price $w$. Sequentially, making the first derivative of the profits of the manufacturer be zero, we can get the optimal wholesale price. All equilibrium results are summarized in Table 1.

\section{E Structure versus $P$ Structure}

Before the comparison analysis, we summarized the equilibrium results for the single channel structure in Table 1. And all the proofs of the propositions are shown in the Appendix.

\subsection{Comparison of Equilibrium Decisions and Profits}

Proposition 1. The equilibrium wholesale price and the retail price in the traditional channel satisfy $w^{E}<w^{N}$ and $w^{P}<w^{N}$ and $p_{R}^{E}<p_{R}^{N}$ and $p_{R}^{P}<p_{R}^{N}$.

Proposition 1 shows that the competition effect of channels in the dual-channel structure makes the sale price of the physical channel lower than that in the single channel structure. In order to ease the channel conflict, the manufacturer decreases the wholesale price to the traditional retailer.

Proposition 2. The equilibrium wholesale price satisfies $w^{E}<$ $w^{P}$, if the commission rate of the platform $\alpha<\widehat{\alpha}_{1} \equiv\left(3 \delta^{2}-7 \delta+\right.$ $4) / \delta(\delta+1)$.

In dual-channel structure, the profit of the manufacturer comes from two channels. In $E$ Structure, the marginal profits which the manufacturer gets from both channels are unit wholesale price. In $P$ Structure, the marginal profit which the manufacturer gets from the electronic channel equals the unit price minus the unit pay on commission. Thus, the commission rate $\alpha$ is a significant factor for the manufacturer weighing the traditional channel and the electronic channel. The higher the commission rate $\alpha$ is, the more the commission is paid and the lower the marginal profit of the manufacturer is gotten from the electronic channel. It means that when the commission rate $\alpha$ is high sufficiently, the manufacturer is more dependent on the traditional channel than on the electronic channel.

Proposition 3. The equilibrium retail price in traditional channel satisfies $p_{R}^{E}<p_{R}^{P}$, if the commission rate of the platform $\alpha<\widehat{\alpha}_{2} \equiv\left(3 \delta^{4}-28 \delta^{3}+87 \delta^{2}-104 \delta+32\right) / \delta\left(8+\delta^{3}-6 \delta^{2}+5 \delta\right)$. The equilibrium retail price in electronic channel satisfies $p_{D}^{E}>p_{D}^{P}$. 
Proposition 3 shows that, in $P$ Structure, $\alpha$ is the cost of the manufacturer adding the electronic channel. The higher the cost is, the higher the retail price in electronic channel is and the higher the retail price is in traditional channel which competes with the electronic channel. When the commission rate $\alpha$ exceeds the threshold $\widehat{\alpha}_{2}$, the retail price in $P$ Structure is higher than that in $E$ Structure.

Proposition 4. The equilibrium sales volume in the two structures satisfies $Q_{R}^{E}>Q_{R}^{P}$ and $Q_{D}^{E}<Q_{D}^{P}$.

Proposition 4 states that, for the traditional channel, the equilibrium sales volume has more advantages in $E$ Structure. The reason is that, in $E$ Structure, the traditional retailer's rival of the game is the e-tailer and consumer perception of the electronic channels is smaller than the traditional channels. In $P$ Structure, the traditional retailer's rival of the game is the manufacturer, but the traditional retailer has less power in the game as the manufacturer is the game leader. For the electronic channel, the lower retail price in $P$ Structure leads to bigger sales volume.

Proposition 5. The equilibrium profits of traditional retailer in the two structures satisfy $\pi_{T R}^{E}>\pi_{T R}^{P}$, and the equilibrium profits of the manufacturer in the two structures satisfy $\pi_{M}^{E} \leq$ $\pi_{M}^{P}$, if the commission rate of the platform $\alpha<\widehat{\alpha}_{3} \equiv(32-80 \delta+$ $\left.83 \delta^{2}-28 \delta^{3}+3 \delta^{4}\right) / \delta\left(32+\delta-6 \delta^{2}+\delta^{3}\right)$.

Proposition 5 illustrates that the traditional retailer earns more money in $E$ Structure than in $P$ Structure. The reason is that, in $E$ Structure, the traditional retailer's rival of the game is the e-tailer and the consumer perception of the electronic channel is lower than the traditional channel. However, in $P$ Structure, the traditional retailer's rival of the game is the manufacturer, and the traditional retailer has less power in the game as the manufacturer is the game leader. For the manufacturer, in $P$ Structure, the commission rate $\alpha$ is the cost spent on the electronic channel. When $\alpha<\widehat{\alpha}_{3}$, the manufacturer's marginal profit in $P$ Structure is higher than in $E$ Structure, so the manufacturer will prefer $P$ Structure. When $\alpha>\widehat{\alpha}_{3}$, the case is opposite.

Proposition 6. (1) In E Structure, when $2-\sqrt{2}<\delta<3-\sqrt{5}$, the manufacturer and the traditional retailer will form a "winwin" situation; when $3-\sqrt{5}<\delta<1$, the manufacturer and the traditional retailer will form a "win-lose" situation; when $0<\delta<2-\sqrt{2}$, the manufacturer and the traditional retailer will form a "lose-win" situation.

(2) In $P$ Structure, when $\alpha \leq(3+2 \delta) / 9$, the manufacturer and the traditional retailer will form a "win-lose" situation. Otherwise, the manufacturer and the traditional retailer will form a "lose-lose" situation.

Proposition 6 illustrates that, in $E$ Structure, when the consumer perception of the electronic channel is modest, the manufacturer and the traditional retailer form a "winwin" situation, and when the consumer perception of the electronic channel is high, $E$ Structure harms the interests of the traditional retailer. Finally, when the consumer perception of the electronic channel is low, rational manufacturer will not adopt $E$ Structure to develop the electronic channel. Dual-channel supply chain strategy always harms the interests of the traditional retailer if the manufacturer adopts $P$ Structure. At the same time, the manufacturer will consider the relationship of the commission rate and the consumer perception of the electronic channel. If the commission rate relative to the consumer electronics channel perception is too high, rational manufacturers will not adopt $P$ Structure to develop the electronic channel.

5.2. Calculation of Consumer Surplus and Social Welfare. Referencing the study of Örsdemir et al. [26], we denote the consumer surplus and the social welfare as

$$
\begin{aligned}
\mathrm{CS} & =\int_{1-\mathrm{Q}_{R}-\mathrm{Q}_{D}}^{1-\mathrm{Q}_{R}}\left(\delta v-p_{D}\right) d v+\int_{1-\mathrm{Q}_{R}}^{1}\left(v-p_{R}\right) d v, \\
\mathrm{SW} & =\int_{1-\mathrm{Q}_{\mathrm{R}}-\mathrm{Q}_{D}}^{1-\mathrm{Q}_{R}} \delta v d v+\int_{1-\mathrm{Q}_{R}}^{1} v d v .
\end{aligned}
$$

According to the calculation formulas and the equilibrium results above, we can easily get the consumer surplus and the social welfare in the single channel structure as $\mathrm{CS}^{N}=$ $1 / 32$ and $\mathrm{SW}^{N}=7 / 32$. At the same time, we can get the consumer surplus and the social welfare in $E$ Structure and in $P$ Structure, as follows:

$$
\begin{aligned}
& \mathrm{CS}^{E}=\frac{64-140 \delta+153 \delta^{2}-75 \delta^{3}+15 \delta^{4}-\delta^{5}}{32(4-\delta)^{2}}, \\
& \mathrm{CS}^{P} \\
& =\frac{\left(16+52 \delta-52 \delta^{2}+9 \delta^{3}\right)+4\left(4+2 \delta-\delta^{2}\right) \delta \alpha+4 \delta^{2} \alpha^{2}}{8(8-3 \delta+\delta \alpha)^{2}}, \\
& \mathrm{SW}^{E}=\frac{192-212 \delta+111 \delta^{2}-5 \delta^{3}-7 \delta^{4}+\delta^{5}}{32(4-\delta)^{2}}, \\
& \mathrm{SW}^{P} \\
& =\frac{\left(112-108 \delta+20 \delta^{2}+\delta^{3}\right)+8(8-3 \delta) \delta \alpha+4 \delta^{2} \alpha^{2}}{8(8-3 \delta+\delta \alpha)^{2}} .
\end{aligned}
$$

The expressions of the consumer surplus and the social welfare in $E$ Structure and in $P$ Structure are complicated. Therefore, we analyze them using the numerical analysis in the next section.

\section{Numerical Analysis}

In this section, in order to verify the aforementioned analysis and to study the change of the consumer surplus and the social welfare in different supply chain structures, we conduct several numerical studies. And we set the parameters variation range as shown in Table 2.

The changes of the profits, the consumer surplus, and the social welfare with the parameter $\alpha$ and $\delta$ are shown in Figures 2-5. 
TABLE 2: The intervals of parameters.

\begin{tabular}{lc}
\hline Parameter & Variation range \\
\hline$\alpha$ & {$[0.1,0.2,0.3,0.4]$} \\
$\delta$ & $0 \sim 1$ \\
\hline
\end{tabular}

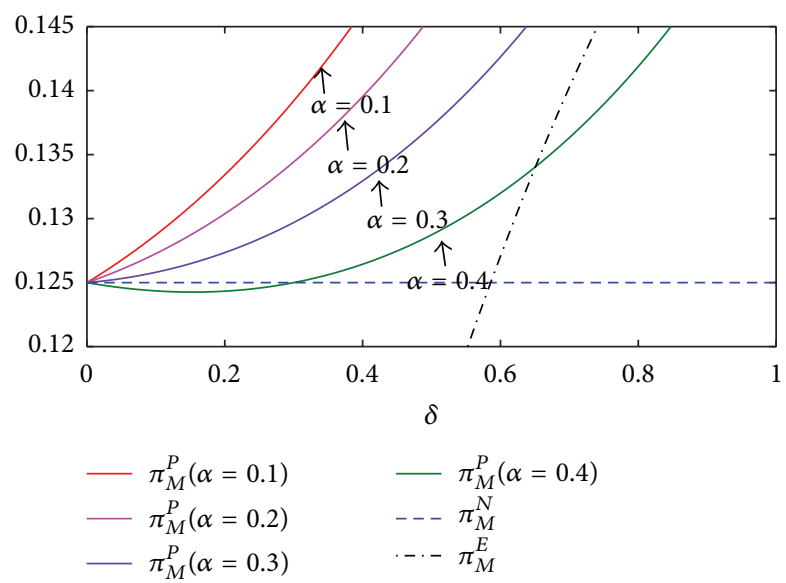

FIGURE 2: $\pi_{M}$ in different supply chain structures.

Figure 2 depicts the change of the manufacture's profit $\pi_{M}$ according to the coefficient of electronic channel perception $\delta$ and platform commission ratio $\alpha$ in different supply chain structures. It shows that the manufacturer earns more money in $E$ Structure than single channel structure only when the parameter $\delta$ is sufficiently high. In $P$ Structure, the profits of the manufacturer decrease with the increasing of $\alpha$. And the manufacturer only benefits in the condition of the platform commission ratio $\alpha$ within a certain scope of the function of $\delta$. At the same time, we find out that when the parameter $\delta$ is high, the manufacturer gets better profits in $E$ Structure than in $P$ Structure.

Figure 3 depicts the change of the traditional retailer's profit $\pi_{\mathrm{TR}}$ according to the coefficient of electronic channel perception $\delta$ and the platform commission ratio $\alpha$ in different supply chain structures. Figure 3 shows that traditional retailer earns more money in $E$ Structure than that in single channel structure if the electronic channel perception $\delta$ is not high enough. But if the electronic channel is competitive sufficiently, $E$ Structure will reduce the profits of the traditional retailer. However, $P$ Structure always hurts the profits of the traditional retailer. Finally, we can indicate that the profit of traditional retailer in $P$ Structure is always higher than that in $E$ Structure with the same parameter $\delta$.

Combining Figure 2 with Figure 3, we can find that, in $E$ Structure, when the parameter $\delta$ is within a certain scope, a "win-win" situation for the manufacturer and the traditional retailer can be formed. On the contrary, in $P$ Structure this "win-win" situation cannot be formed. Nevertheless, once the parameter $\delta$ is beyond the scope, both of dual-channel supply chain structures damage the interests of the traditional retailers.

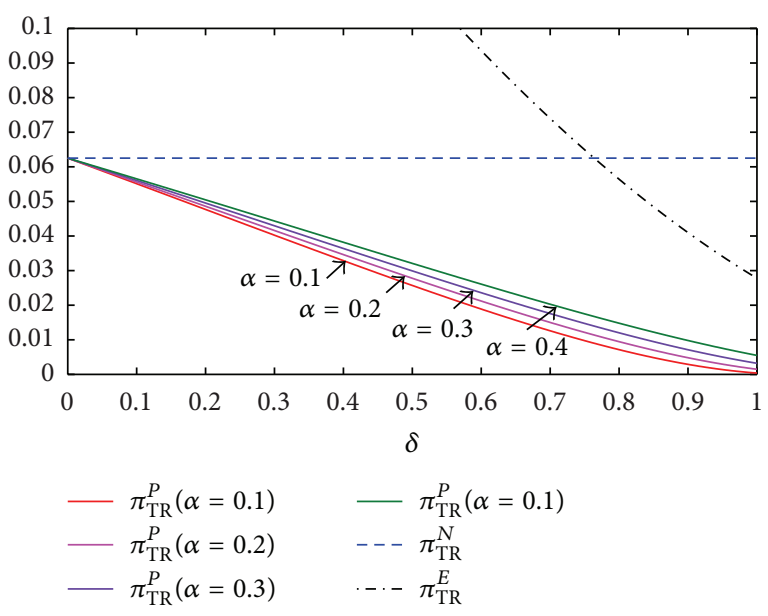

FIGURE 3: $\pi_{\mathrm{TR}}$ in different supply chain structures.

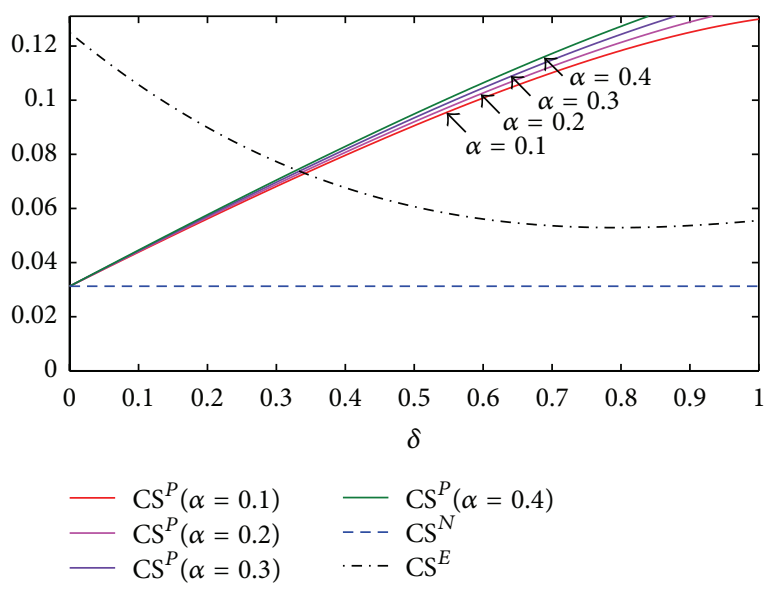

FIGURE 4: Consumer surplus in different supply chain structures.

Figures 4 and 5 depict the change of the consumer surplus and the social welfare according to the coefficient of electronic channel perception $\delta$ and the platform commission ratio $\alpha$ in different supply chain structures. As the figures show, the consumer surplus and the social welfare in any dual-channel structure are always bigger than those in the single channel structure. We also find out that when the parameter $\delta$ is low, the consumer surplus and the social welfare in $E$ Structure are bigger than those in $P$ Structure. When the parameter $\delta$ is big, consumer surplus and social welfare in $P$ Structure are higher than those in $E$ Structure and increase with the growth of the parameter $\alpha$. That is because the price in both channels would decrease with increasing of $\alpha$ in $P$ Structure. The meaning is that the surplus value of the products increases for consumers.

\section{Conclusions}

Most companies in China are small and medium enterprises. In this scenario, the majority of manufacturers develop 


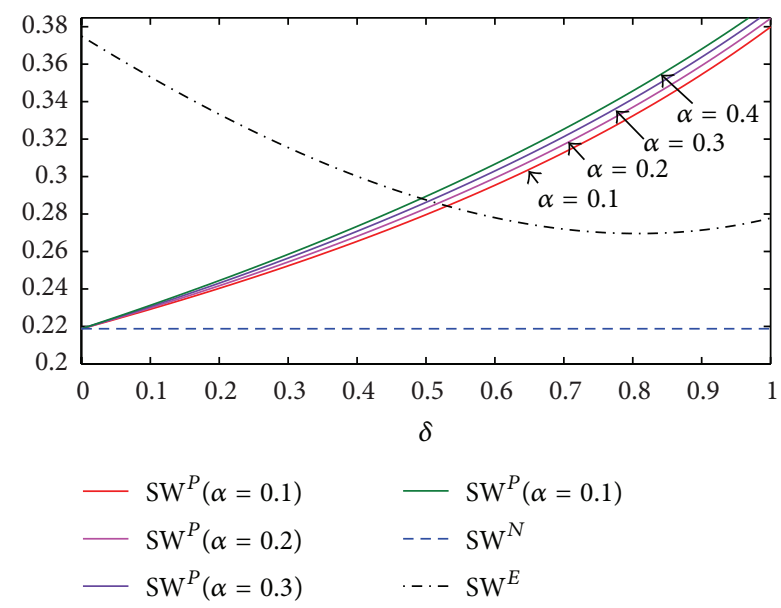

FIGURE 5: Social welfare in different supply chain structures.

the electronic channel via third-party e-commerce platforms. However, the platform owner can act either as an e-tailer or as an intermediary agency that forms two supply chain structures. Utilizing the game theory approach, we compare the two different structures in the paper and summarize some significant conclusions.

The wholesale price and the retail price in both channels of two different structures are always lower than those in the single channel structure. The wholesale price and the retail price in the traditional channel in which dual-channel structure is higher depended on the value of the commission rate $\alpha$. Otherwise, the retail price of the electronic channel in $E$ Structure is always higher than the one in $P$ Structure. It means that the competition is more vehement in $E$ Structure. The important finding is that the manufacturer and the traditional retailer can achieve a "win-win" situation in $E$ Structure if the consumer perception of the electronic channel is modest. In contrast, the dual-channel strategy always damages the interests of the traditional retailers if the manufacturer adopts $P$ Structure. However, the requirement of $\delta$ is higher in $E$ Structure, which means that when the consumer perception of the electronic channel $\delta$ is not high enough, the platform enterprise has no intention to sale products by $E$ Structure and the manufacturer only has the choice of $P$ Structure if the commission rate $\alpha$ is within the ideal range. In the last step we use the method of the numerical analysis to study the change of the consumer surplus and the social welfare in different supply chain structures. The comparison of the results indicates that dualchannel structures are always better for both the consumer surplus and the social welfare than single channel structure, and $E$ Structure is better for the consumer surplus and the social welfare than $P$ Structure when the consumer perception of the electronic channel is low. Oppositely, $P$ Structure is better for the consumer surplus and the social welfare than $E$ Structure when the consumer perception of electronic channel is high.
From this research, we can find that the traditional retailer prefers $E$ Structure when the supply chain develops the electronic channel. And the manufacturer prefers $P$ Structure if the commission rate $\alpha$ is within the ideal range. However, when the consumer perception of the electronic channel $\delta$ is sufficiently high, which means the consumers' acceptability of the electronic channel is also sufficiently high, any dualchannel structure decreases the interest of the traditional retailer. Thus, the traditional retailers should adopt measures such as good service to improve the consumer perception of the traditional channel.

\section{Appendix}

All of the equilibrium results in different supply chain structures used in proofs are gotten in Section 4 , and parameters satisfy the conditions $0<\delta<1$ and $0<\alpha<1$.

Proof of Proposition 1. Comparing the equilibrium wholesale price and sale price in different dual-channel supply chain structures with that in the single channel,

$$
\begin{aligned}
w^{E}-w^{N} & =\frac{\delta(3-\delta)}{4}-\frac{1}{2}=-\frac{(1-\delta)(2-\delta)}{4}<0, \\
w^{P}-w^{N} & =\frac{8-4 \delta+\delta^{2}-2 \delta \alpha}{2(8-3 \delta+\delta \alpha)}-\frac{1}{2} \\
& =-\frac{\delta(1+3 \alpha-\delta)}{2(8-3 \delta+\delta \alpha)}<0, \\
p_{R}^{E}-p_{R}^{N} & =\frac{\left(8+5 \delta-6 \delta^{2}+\delta^{3}\right)}{4(4-\delta)}-\frac{3}{4} \\
& =\frac{\delta(2-\delta)}{4}-\frac{1}{(4-\delta)}<\frac{\delta(2-\delta)}{4}-\frac{1}{4} \\
& =-\frac{(\delta-1)^{2}}{4}<0, \\
p_{R}^{P}-p_{R}^{N} & =\frac{(2-\delta)(6-\delta)}{2(8-3 \delta+\delta \alpha)}-\frac{3}{4}=-\frac{\delta(7-2 \delta+3 \alpha)}{4(8-3 \delta+\delta \alpha)} \\
& <0 .
\end{aligned}
$$

Proof of Proposition 2. Comparing the equilibrium wholesale price in $E$ Structure with that in $P$ Structure,

$$
\begin{aligned}
w^{E}-w^{P} & =\frac{\delta(3-\delta)}{4}-\frac{8-4 \delta+\delta^{2}-2 \delta \alpha}{2(8-3 \delta+\delta \alpha)} \\
& =-\frac{(4-\delta)\left(4+3 \delta^{2}-7 \delta-\delta(1+\delta) \alpha\right)}{4(8-3 \delta+\delta \alpha)} .
\end{aligned}
$$

So we obtain a threshold value

$$
\widehat{\alpha}_{1} \equiv \frac{3 \delta^{2}-7 \delta+4}{\delta(\delta+1)} .
$$


Proof of Proposition 3. (1) Comparing the equilibrium retail price of the traditional channel in $E$ Structure with that in $P$ Structure,

$$
p_{R}^{E}-p_{R}^{P}=-\frac{\left(32-104 \delta+84 \delta^{2}-28 \delta^{3}+3 \delta^{4}-\left(8 \delta+5 \delta^{2}-6 \delta^{3}+\delta^{4}\right) \alpha\right)}{4(4-\delta)(8-3 \delta+\delta \alpha)}
$$

We obtain a threshold value

$$
\widehat{\alpha}_{2} \equiv \frac{3 \delta^{4}-28 \delta^{3}+87 \delta^{2}-104 \delta+32}{\delta\left(8+\delta^{3}-6 \delta^{2}+5 \delta\right)} .
$$

(2) Comparing the equilibrium retail price of the electronic channel in $E$ Structure with in $P$ Structure,

$$
\begin{aligned}
p_{D}^{E}-p_{D}^{P} & =\frac{\delta(5-\delta)}{2(4-\delta)}-\frac{\delta(6-\delta)}{2(8-3 \delta+\delta \alpha)} \\
& =\frac{\delta\left(16-13 \delta+5 \delta \alpha+\delta^{2}(2-\alpha)\right)}{2(4-\delta)(8-3 \delta+\delta \alpha)}>0 .
\end{aligned}
$$

Proof of Proposition 4. Comparing the equilibrium sales volume of the traditional channel and the electronic channel in $E$ Structure with that in $P$ Structure,

$$
\begin{aligned}
Q_{R}^{E}- & Q_{R}^{P} \\
& =\frac{\left(32-40 \delta+21 \delta^{2}-3 \delta^{3}-\left(8 \delta+3 \delta^{2}-\delta^{3}\right) \alpha\right)}{4(4-\delta)(8-3 \delta+\delta \alpha)} \\
& >\frac{32-40 \delta+21 \delta^{2}-3 \delta^{3}-\left(8 \delta+3 \delta^{2}-\delta^{3}\right)}{4(4-\delta)(8-3 \delta+\delta \alpha)} .
\end{aligned}
$$

Proof of Proposition 5. Comparing the equilibrium profits of the traditional retailer and the manufacturer in $E$ Structure with that in $P$ Structure,
Denote a function as

$$
g_{1}(\delta)=32-40 \delta+21 \delta^{2}-3 \delta^{3}-\left(8 \delta+3 \delta^{2}-\delta^{3}\right)
$$

Because $\partial g_{1}(\delta) / \partial \delta=-6 \delta^{2}+36 \delta-48<0, \partial^{2} g_{1}(\delta) / \partial \delta^{2}=$ $-12 \delta+36>0$.

We can obtain $g(\delta)>g(1)=1>0$, and then $Q_{R}^{E}-Q_{R}^{P}>0$ :

$$
\begin{aligned}
Q_{D}^{E}-Q_{D}^{P} & =\frac{\left(5 \delta-\delta^{2}-2\right)}{4(4-\delta)}-\frac{6-\delta}{2(8-3 \delta+\delta \alpha)} \\
& <\frac{\left(5 \delta-\delta^{2}-2\right)}{4(4-\delta)}-\frac{6-\delta}{2(8-3 \delta+\delta)} \\
& =-\frac{\left(8-6 \delta+\delta^{2}\right)}{4(4-\delta)}<0 .
\end{aligned}
$$

$$
\pi_{\mathrm{TR}}^{E}-\pi_{\mathrm{TR}}^{P}=\frac{\left(32-40 \delta+21 \delta^{2}-3 \delta^{3}-\left(8 \delta+3 \delta^{2}-\delta^{3}\right) \alpha\right)\left(96-120 \delta+37 \delta^{2}-3 \delta^{3}+\left(24 \delta-11 \delta^{2}+\delta^{3}\right) \alpha\right)}{16(4-\delta)^{2}(8-3 \delta+\delta \alpha)^{2}} .
$$

We get $\left(32-40 \delta+21 \delta^{2}-3 \delta^{3}-\left(8 \delta+3 \delta^{2}-\delta^{3}\right) \alpha\right)>0$ in proof of Proposition 4.

It is obvious that $\left(96-120 \delta+37 \delta^{2}-3 \delta^{3}+\left(24 \delta-11 \delta^{2}+\right.\right.$ $\left.\left.\delta^{3}\right) \alpha\right)>96-120 \delta+37 \delta^{2}-3 \delta^{3}$.
Denote a function as

$$
g_{2}(\delta)=96-120 \delta+37 \delta^{2}-3 \delta^{3} .
$$

Because $\partial g_{2}(\delta) / \partial \delta=-120+74 \delta-9 \delta^{2}<0, \partial^{2} g_{2}(\delta) / \partial \delta^{2}=$ $-18 \delta+74>0$.

We can obtain $g_{2}(\delta)>g_{2}(1)>0$, and then $\pi_{\mathrm{TR}}^{E}-\pi_{\mathrm{TR}}^{P}>0$.

$$
\pi_{M}^{E}-\pi_{M}^{P}=-\frac{\left(32-80 \delta+83 \delta^{2}-28 \delta^{3}+3 \delta^{4}-\left(32 \delta+\delta^{2}-6 \delta^{3}+\delta^{4}\right) \alpha\right)}{8(4-\delta)(8-3 \delta+\delta \alpha)} .
$$


We obtain the threshold value

$$
\widehat{\alpha}_{3} \equiv \frac{32-80 \delta+83 \delta^{2}-28 \delta^{3}+3 \delta^{4}}{\delta\left(32+\delta-6 \delta^{2}+\delta^{3}\right)} .
$$

Proof of Proposition 6. (1) Comparing the profits of the manufacturer and the traditional retailer in $E$ Structure with that in the single channel structure,

$$
\begin{aligned}
\pi_{M}^{E}-\pi_{M}^{N} & =\frac{\delta(3-\delta)^{2}}{8(4-\delta)}-\frac{1}{8} \\
& =\frac{(2-\delta)\left((\delta-2)^{2}-2\right)}{8(4-\delta)}, \\
\pi_{\mathrm{TR}}^{E}-\pi_{\mathrm{TR}}^{N} & =\frac{\left(\delta^{2}-7 \delta+8\right)^{2}}{16(4-\delta)^{2}}-\frac{1}{16} \\
& =\frac{(2-\delta)(6-\delta)\left((\delta-3)^{2}-5\right)}{16(4-\delta)^{2}}
\end{aligned}
$$

In summary, we obtain $\pi_{M}^{E}>\pi_{M}^{N}$ and $\pi_{\mathrm{TR}}^{E}>\pi_{\mathrm{TR}}^{N}$, when 2$\sqrt{2}<\delta<3-\sqrt{5} ; \pi_{M}^{E}>\pi_{M}^{N}$ and $\pi_{\mathrm{TR}}^{E}<\pi_{\mathrm{TR}}^{N}$, when $3-\sqrt{5}<$ $\delta<1 ; \pi_{M}^{E}<\pi_{M}^{N}$ and $\pi_{\mathrm{TR}}^{E}>\pi_{\mathrm{TR}}^{N}$, when $0<\delta<2-\sqrt{2}$.

(2) Comparing the profit of the manufacturer and the traditional retailer in $P$ Structure with that in the single channel structure,

$$
\begin{aligned}
\pi_{M}^{p}-\pi_{M}^{N} & =\frac{4+\delta^{2}-4 \delta \alpha}{4(8-3 \delta+\delta \alpha)}-\frac{1}{8} \\
& =\frac{\delta(3+2 \delta-9 \alpha)}{8(8-3 \delta+\delta \alpha)} \\
\pi_{\mathrm{TR}}^{P}-\pi_{\mathrm{TR}}^{N} & =\frac{(2-2 \delta+\delta \alpha)^{2}}{(8-3 \delta+\delta \alpha)^{2}}-\frac{1}{16} \\
& =-\frac{\delta(5-3 \alpha)(16-11 \delta+5 \delta \alpha)}{16(8-3 \delta+\delta \alpha)^{2}}>0 .
\end{aligned}
$$

In summary, we obtain $\pi_{M}^{p}>\pi_{M}^{N}$ and $\pi_{\mathrm{TR}}^{P}>\pi_{\mathrm{TR}}^{N}$, when $0<$ $\alpha<(3+2 \delta) / 9 ; \pi_{M}^{p}<\pi_{M}^{N}$ and $\pi_{\mathrm{TR}}^{P}>\pi_{\mathrm{TR}}^{N}$, when $(3+2 \delta) / 9<$ $\alpha<1$.

\section{Competing Interests}

The authors declare that they have no competing interests.

\section{References}

[1] S.-H. Chun, B.-D. Rhee, S. Y. Park, and J.-C. Kim, "Emerging dual channel system and manufacturer's direct retail channel strategy," International Review of Economics \& Finance, vol. 20, no. 4, pp. 812-825, 2011.

[2] L. Ma, R. Zhang, S. Guo, and B. Liu, "Pricing decisions and strategies selection of dominant manufacturer in dual-channel supply chain," Economic Modelling, vol. 29, no. 6, pp. 2558-2565, 2012.
[3] V. Abhishek, K. Jerath, and Z. J. Zhang, "Agency selling or reselling? channel structures in electronic retailing," SSRN Electronic Journal, 2015.

[4] W.-Y. K. Chiang, D. Chhajed, and J. D. Hess, "Direct marketing, indirect profits: a strategic analysis of dual-channel supplychain design," Management Science, vol. 49, no. 1, pp. 1-20, 2003.

[5] S. R. Croom, "The impact of e-business on supply chain management: an empirical study of key developments," International Journal of Operations and Production Management, vol. 25, no. 1, pp. 55-73, 2005.

[6] M. R. Baye, "Price competition between pure play vs. bricksand-clicks e-tailers: analytical model and empirical analysis," Social Science Electronic Publishing, vol. 11, pp. 29-61, 2002.

[7] S. Y. Park and H. T. Keh, "Modelling hybrid distribution channels: a game-theoretic analysis," Journal of Retailing and Consumer Services, vol. 10, no. 3, pp. 155-167, 2003.

[8] Q. Lu and N. Liu, "Effects of e-commerce channel entry in a two-echelon supply chain: a comparative analysis of singleand dual-channel distribution systems," International Journal of Production Economics, vol. 165, pp. 100-111, 2015.

[9] B. Li, P. Chen, Q. Li, and W. Wang, "Dual-channel supply chain pricing decisions with a risk-averse retailer," International Journal of Production Research, vol. 52, no. 23, pp. 7132-7147, 2014.

[10] L. Li, J. He, and Q. Shi, "Dual-channel pricing strategies for small and medium-sized enterprises," International Journal of Services, Technology and Management, vol. 20, no. 1-3, pp. 4770, 2014.

[11] E. Cao, "Coordination of dual-channel supply chains under demand disruptions management decisions," International Journal of Production Research, vol. 52, no. 23, pp. 7114-7131, 2014.

[12] J. Chen, H. Zhang, and Y. Sun, "Implementing coordination contracts in a manufacturer stackelberg dual-channel supply chain," Omega, vol. 40, no. 5, pp. 571-583, 2012.

[13] X. U. Guang ye, B. Dan, and J. Xiao, "Study on dual-channel supply chain coordination with new revenue-sharing contract," Chinese Journal of Management Science, vol. 18, no. 6, pp. 59-64, 2010.

[14] J. K. Ryan, D. Sun, and X. Zhao, "Coordinating a supply chain with a manufacturer-owned online channel: a dual channel model under price competition," IEEE Transactions on Engineering Management, vol. 60, no. 2, pp. 247-259, 2013.

[15] A. A. Tsay and N. Agrawal, "Channel conflict and coordination in the E-commerce age," Production \& Operations Management, vol. 13, no. 1, pp. 93-110, 2004.

[16] J. Xiao, B. Dan, and X.-M. Zhang, "Service cooperation pricing strategy between manufacturers and retailers in dual-channel supply chain," System Engineering Theory \& Practice, vol. 30, no. 12, pp. 2203-2211, 2010.

[17] Z.-Y. Zhang, H.-J. Li, L. Yang, and Y.-Q. Shi, "Dual-channel coordination strategies on advertising cooperation based on differential game," Control \& Decision, vol. 29, no. 5, pp. 873879, 2014.

[18] J.-C. Rochet and J. Tirole, "Two-sided markets: a progress report," Rand Journal of Economics, vol. 37, no. 3, pp. 645-667, 2006.

[19] D. Sen, "Fee versus royalty reconsidered," Games \& Economic Behavior, vol. 53, no. 1, pp. 141-147, 2005.

[20] X. H. Wang, "Fee versus royalty licensing in a cournot duopoly model," Economics Letters, vol. 60, no. 1, pp. 55-62, 1998. 
[21] J. Muthers and S. Wismer, "Why do platforms charge proportional fees? commitment and seller participation," SSRN'S Electronic Journals, 2013.

[22] Z. Wang and J. Wright, Ad-Valorem Platform Fees, Indirect Taxes and Efficient Price Discrimination, Social Science Electronic Publishing, 2015.

[23] A. Hagiu, "Merchant or two-sided platform?" Review of Network Economics, vol. 6, no. 2, pp. 115-133, 2007.

[24] B. Mantin, H. Krishnan, and T. Dhar, "The strategic role of third-party marketplaces in retailing," Production and Operations Management, vol. 23, no. 11, pp. 1937-1949, 2014.

[25] B. Jiang, K. Jerath, and K. Srinivasan, "Firm strategies in the 'mid tail' of platform-based retailing," Marketing Science, vol. 30, no. 5, pp. 757-775, 2011.

[26] A. Örsdemir, E. Kemahlığlu-Ziya, and A. K. Parlaktürk, "Competitive quality choice and remanufacturing," Production \& Operations Management, vol. 23, no. 1, pp. 48-64, 2014. 


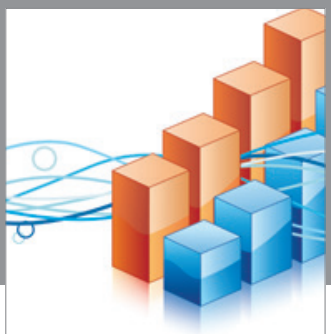

Advances in

Operations Research

vatem alat4

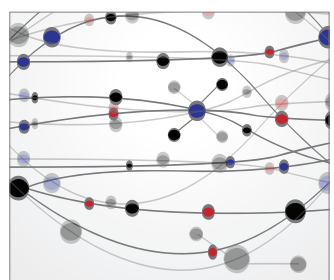

\section{The Scientific} World Journal
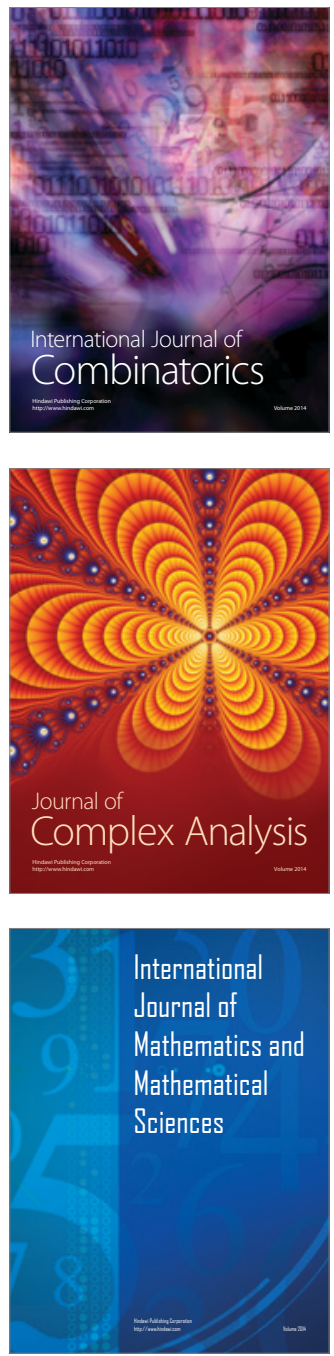
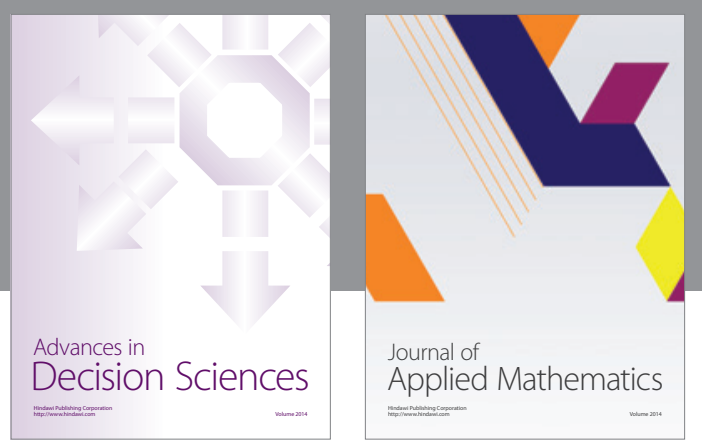

Algebra

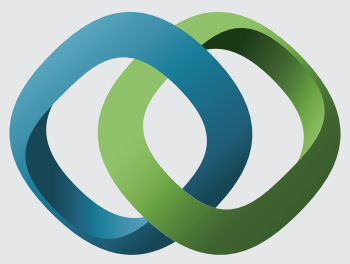

\section{Hindawi}

Submit your manuscripts at

http://www.hindawi.com
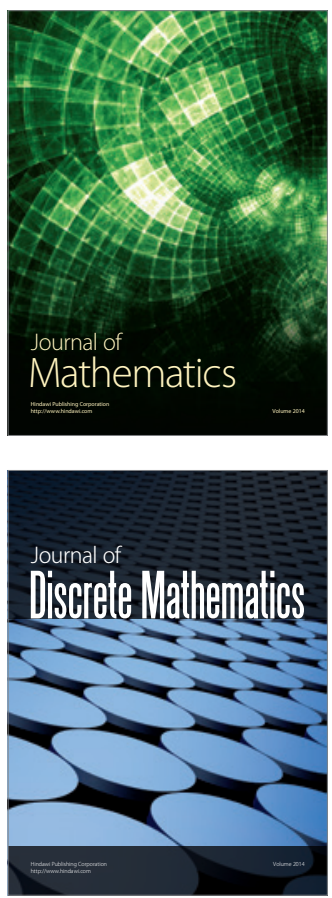

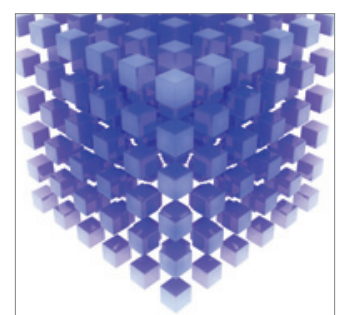

Mathematical Problems in Engineering
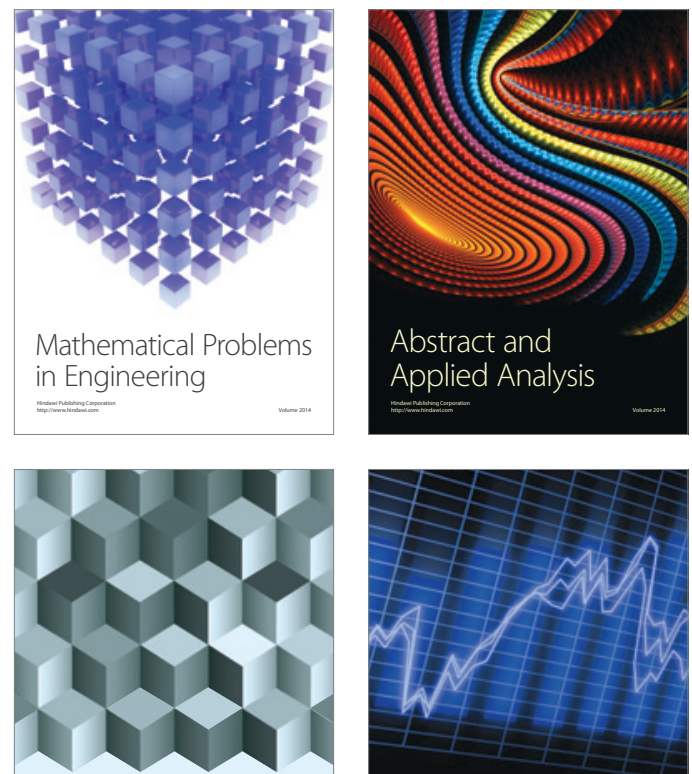

Journal of

Function Spaces

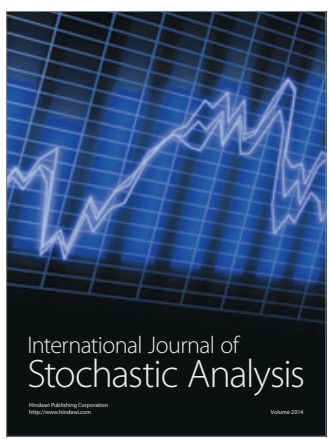

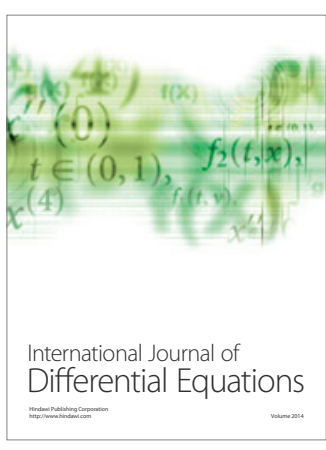
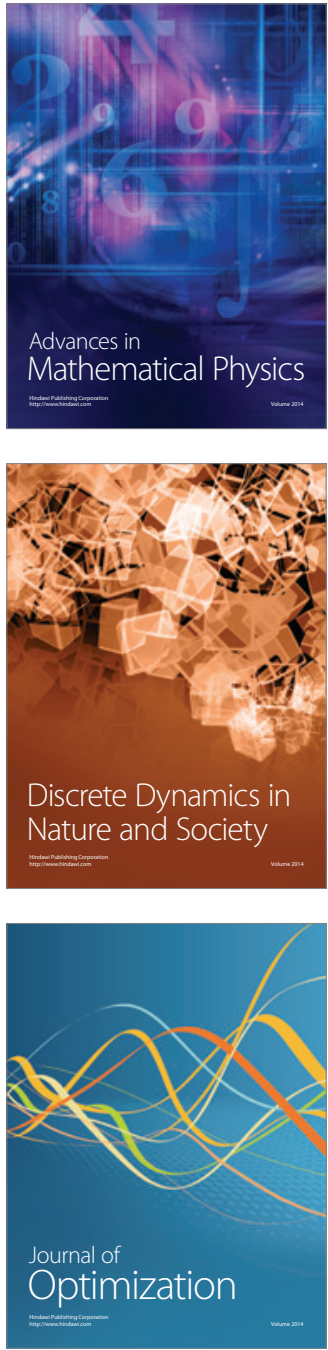